\title{
Article \\ Experimental Characterization of Low-Speed Passive Discharge Losses of a Flywheel Energy Storage System
}

\author{
Miles Skinner and Pierre Mertiny *(D) \\ Department of Mechanical Engineering, University of Alberta, 9211-116 St., Edmonton, AB T6G 1H9, Canada; \\ maskinne@ualberta.ca \\ * Correspondence: pmertiny@ualberta.ca
}

Citation: Skinner, M.; Mertiny, P. Experimental Characterization of Low-Speed Passive Discharge Losses of a Flywheel Energy Storage System. Appl. Mech. 2021, 2, 1-15. https://doi.org/10.3390/ applmech2010001

Received: 9 November 2020 Accepted: 8 January 2021 Published: 12 January 2021

Publisher's Note: MDPI stays neutral with regard to jurisdictional clai$\mathrm{ms}$ in published maps and institutional affiliations.

Copyright: (C) 2021 by the authors. Licensee MDPI, Basel, Switzerland. This article is an open access article distributed under the terms and conditions of the Creative Commons Attribution (CC BY) license (https:// creativecommons.org/licenses/by/ $4.0 /)$.

\begin{abstract}
Flywheel energy storage has a wide range of applications in energy grids and transportation. The adoption of high-performance components has made this technology a viable alternative for substituting or complementing other storage devices. Flywheel energy storage systems are subject to passive discharge attributed primarily to electrical machine losses, bearing rolling friction, and aerodynamic drag of the flywheel rotor. In the present study, measurements are presented for complete discharge experiments using a flywheel system featuring a vacuum enclosure. Best-fit equations were applied to the test data and compared to analytical models. Analysis of the best-fit equations indicates that they may serve as empirical models for approximating passive discharge under given conditions. Bearing losses, which varied linearly with velocity but were otherwise unaffected throughout the experiments, were larger than aerodynamic drag at low air pressures and low velocities. Aerodynamic drag became significant as velocity exceeded approximately $3400 \mathrm{rpm}$. The electrical machine was found to be the most significant source of passive discharge at all velocities and pressures. Based on these findings, it is recommended to maintain a low-pressure environment in the flywheel enclosure and to decouple the electrical machine from the rotor whenever possible to eliminate associated losses.
\end{abstract}

Keywords: flywheel energy storage; passive discharge; experimental characterization

\section{Introduction}

Due to the intermittent behavior of wind and solar power generation, significant energy storage capacity is required to satisfy demand. Inconsistent power generation presents challenges in load balancing, electric grid reliability, and power quality. Energy storage systems are anticipated to provide the solution to these issues but are far underutilized and lacking in diversity. In the United States, only $2.5 \%$ of the delivered electrical energy uses energy storage as a critical component in maintaining a reliable supply of energy, while other leading countries are closer to $10 \%$ and $15 \%$, Germany and Japan, respectively. Further, the vast majority of storage capacity is in pumped hydroelectric, with compressed air and electrochemical batteries lagging significantly behind [1,2].

Flywheel energy storage systems (FESSs) have been implemented in electric grids to reduce power spikes, provide frequency regulation, improve power quality, and serve as uninterrupted power supply (UPS) systems due to several advantageous characteristics of FESS technology. This includes high charge and discharge rates, lifetimes ranging from 10 to 20 years, and high specific energy [3]. Further, they do not experience depth of discharge effects and have a relatively high cycle efficiency-up to $95 \%$ depending on the electrical components [2].

Even though FESS have a wide variety of application across many industries, the technology is still developing. Many current studies into FESSs have focused on maximizing energy density, storage time, or other performance metrics using specialized equipment, such as high-performance vacuum pumps and superconducting magnetic bearings (SMB). 
These efforts enabled high or ultra-high vacuum levels and low passive discharge bearings $[4,5]$. While such vacuum levels and bearings are correctly judged to maximize the system performance, it is not, currently, an economically feasible approach to widening the adaptation of FESSs into areas such as transportation, home energy, microgrid and small-scale commercial systems, given the additional cost, electrically and financially, of operating this equipment.

Low-cost, small-scale FESSs present several unique challenges that do not appear in utility-scale systems. Mechanical bearings, for instance, are the most practicable choice for low-cost FESSs as magnetic bearings, especially active or SMB, are likely to hinder a viable design. Low-cost FESSs cannot afford the necessary equipment to reach the vacuum level discussed in [5] or employ the SMB discussed by Coombs et al. [6] and Xia et al. [7]. It is, therefore, desirable to operate low-cost FESSs at weaker vacuum levels and with mechanical bearings. Due to these limitations, passive discharge in low-cost systems are higher relative to the high-performance systems discussed above. While it is important to recognize the contributions of previous studies $[4,8]$, research focusing specifically on low-cost FESSs passive discharge losses are rare. The present study characterizes these losses by first gaining a better understanding of each primary passive discharge source and then experimentally isolating each source and analyzing their characteristics.

\section{Experimental Setup}

The experimental study was performed using the custom-built flywheel energy storage device shown in Figure 1. This FESS is a scaled-down technology demonstrator for an energy storage unit with a nominal capacity of $1.0 \mathrm{kWh}$ for a regenerative braking application in light-rail transit service. Specifically, the rotor shown in Figure 1 is scaled to $24 \%$ in axial height while having the same outer rotor diameter as the full-size design. While the design top speed of the rotor is 30,000 rpm $\left(3141 \mathrm{sec}^{-1}\right)$, the device was herein operated at lower speeds for safety. The primary components of this FESS, i.e., the housing, the rotor, bearings, and electrical machine (motor/generator unit), as well as the instrumentation and ancillary equipment, are described in the following.

\subsection{Housing}

The flywheel housing has three main functions: (i) providing mounting surfaces for the electrical machine, bearing rotor assembly, instrumentation, and any other necessary components; (ii) maintaining the rotor and other rotating components in a vacuum environment; and (iii) serving as a safety feature in the event of a catastrophic rotor or bearing failure. The housing shown in Figure 1 is constructed from a top and bottom aluminum plate providing all the mounting surfaces and a $30 \mathrm{~mm}$ thick cylindrical steel burst ring for safety. The bottom plate has a two-part bearing fixture, seen as the two connected parts surrounding the bottom bearing assembly, consisting of a radial bearing and a magnetic thrust bearing. The top plate holds a single piece fixture with the upper radial bearing, as well as the electrical machine and instruments.

\subsection{Rotor}

The flywheel rotor is constructed as a press-fit assembly from three components, i.e., two composite rims and an aluminum hub. This multi-rim design increases the specific energy density of the rotor and reduces material cost [9-11], which are key objectives for low-cost FESS. The rotor total mass and maximum radius are $7.75 \mathrm{~kg}$ and $200 \mathrm{~mm}$, respectively. The outer rim is made of carbon-fiber-reinforced epoxy polymer (CFRP). The inner rim is composed of aramid-fiber-reinforced epoxy polymer (AFRP). The hub is constructed from aluminum. The hub features an integrated shaft with radial bearing mount surfaces on the top and bottom, a magnetic thrust bearing mount threaded into the bottom, and a magnetic coupling post at the top. Basic properties for the primary rotor components are summarized in Table 1. 


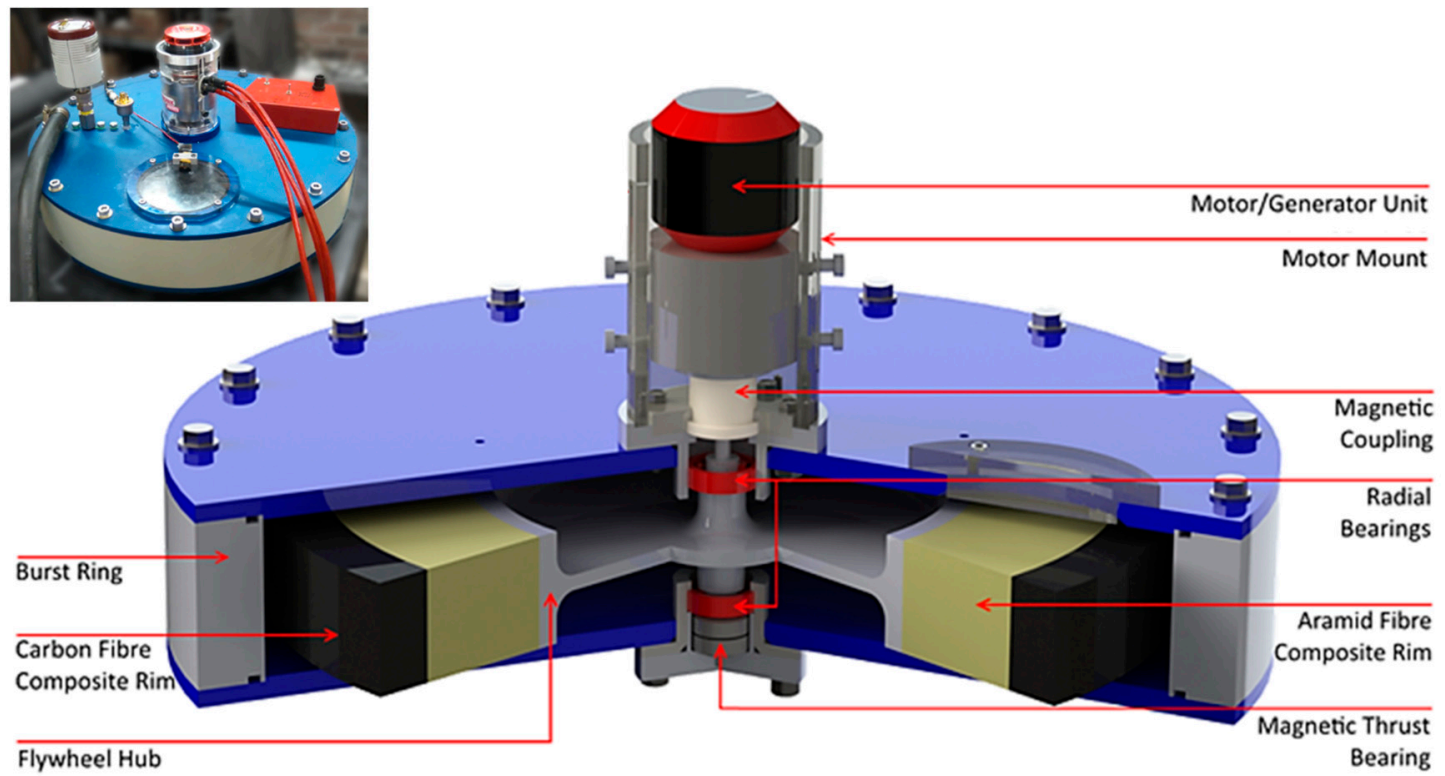

Figure 1. Flywheel energy storage device used in this study: cut-away view with labeled primary components and inset showing a photograph of the actual device.

Table 1. Flywheel rotor dimensions and material properties.

\begin{tabular}{ccc}
\hline Component $^{\mathbf{1}}$ & Inner Radius & Density \\
\hline Outer CFRP rim & $0.170 \mathrm{~m}$ & $1569 \mathrm{~kg} / \mathrm{m}^{3}$ \\
Inner AFRP rim & $0.110 \mathrm{~m}$ & $1380 \mathrm{~kg} / \mathrm{m}^{3}$ \\
Aluminum hub rim & $0.100 \mathrm{~m}$ & $2700 \mathrm{~kg} / \mathrm{m}^{3}$ \\
\hline
\end{tabular}

${ }^{1}$ Axial height of rims and hub is $0.050 \mathrm{~m}$.

\subsection{Bearings}

A detailed cross-section view of the bearing system is shown in Figure 2. The rotor is supported axially by a trust bearing consisting of two permanent neodymium magnets with opposing magnetic fields and radially by two 6002 full silicon nitride deep groove ball bearings (Bearings Canada, Concord, ON, Canada) with a PTFE cage. The upper bearing assembly has a series of spring washers pressing on the outer bearing race to create a small, approximately $60 \mathrm{~N}$, axial preload, which is important to stabilize the upper bearing and minimize vibrational loading.

The magnets of the thrust bearing, item 6 in Figure 2, support the majority but not all of the rotor's weight to reduce rolling friction in the ball bearings. Akin to the function of the spring washers, an axial force corresponding to approximately $30 \%$ of the rotor weight is resting on the lower bearing. The axial magnetic bearings are not self-centering, so the resulting instability creates a small radial force that marginally increases frictional losses and cyclic loading on the bearings.

\subsection{Electrical Machine}

The electrical machine is a brushless permanent magnet excited synchronous machine (Pyro 850-50L, Kontronik, Rottenburg-Hailfingen, Germany). The motor has a rated speed of 30,000 rpm, a maximum torque of $2 \mathrm{Nm}$, and maximum power output of $6 \mathrm{~kW}$. Power is supplied to the motor through a Kontronik Kosmik 160LV electronic speed controller, which in turn is powered by an RT2000 power supply outputting $2 \mathrm{~kW}$ at $56 \mathrm{~V}$ (Lucent Technologies Inc., Murray Hill, NJ, USA). The electrical machine, which is running in ambient conditions (i.e., outside the vacuum enclosure), is coupled to the rotor, through 
the housing, via a passive magnetic coupling (see Figure 1). The magnetic coupling is assembled to the housing to provide a vacuum-tight system.

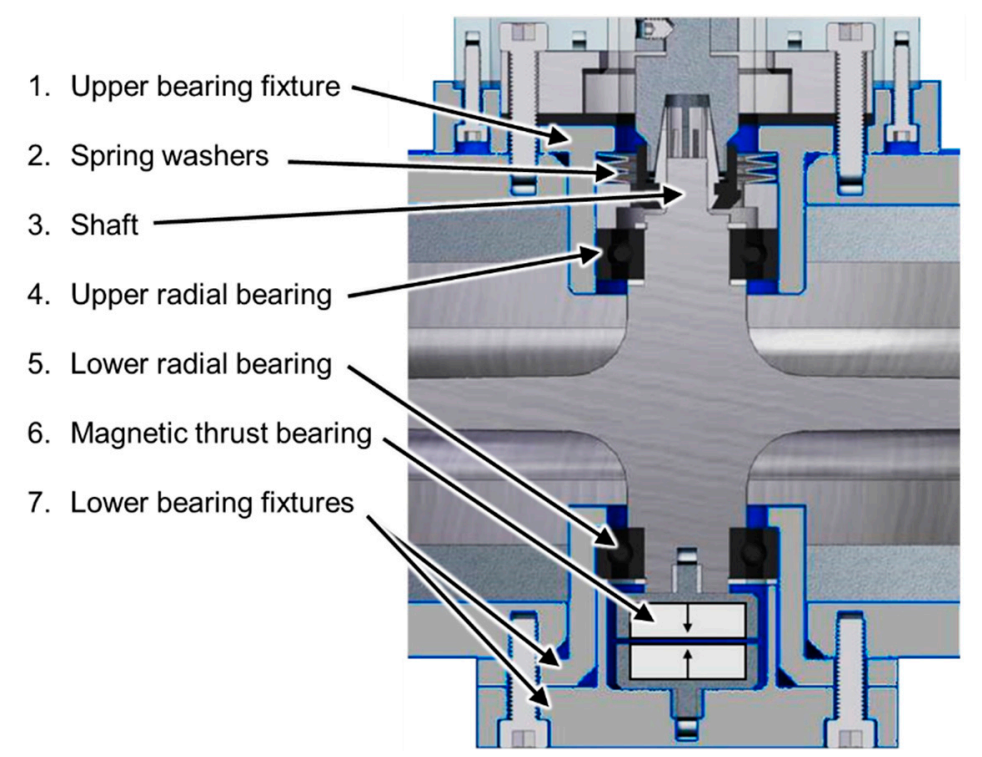

Figure 2. Schematic with labeled components of the upper and lower bearing sub-assemblies.

\subsection{Instrumentation and Ancillary Equipment}

A vacuum is maintained inside the FESS housing operating at or below $133 \mathrm{~Pa}$ (1 Torr). The vacuum system, in combination with the mechanical vacuum pump (Trivac D8A, Leybold, Cologne, Germany), provides an effective pressure range of $4 \mathrm{~Pa}$ to $1.33 \mathrm{kPa}$ that can accurately be maintained within $0.01 \mathrm{~Pa}$ once in equilibrium. Two vacuum sensors are connected to the FESS to continuously monitor the pressure inside the housing during testing. A Baratron vacuum gauge (MKS Instruments, Andover, MA, USA) is used to monitor the pressure from atmospheric pressure $(101.3 \mathrm{kPa})$ to $133.3 \mathrm{~Pa}$, and it is connected to a pressure gauge controller (CA SR5 SP411 11 0, Wika Instruments, Edmonton, $\mathrm{AB}, \mathrm{Canada}$ ). Second, a thermocouple vacuum gauge is used for pressures from $133.3 \mathrm{~Pa}$ to $0.133 \mathrm{~Pa}$ (transducer No.270006 connected to an ionization gauge controller Series 270, Granville Phillips, Longmont, CO, USA). The velocity of the FESS rotor is monitored by a custom-built optical speed sensor that detects ambient light reflecting off a single reflective spot on the rotor. The measurement equipment is connected to a NI USB-6009 data acquisition system (National Instruments, Austin, TX, USA). For this study, the sample rate was $1 \mathrm{~ms}$ with an accuracy of $1 \mathrm{~Hz}$. Data were recorded using a personal computer.

\section{Experimental Methodology}

There are three primary sources of passive discharge losses associated with the operation of the considered FESS: (i) electromotive and mechanical losses in the electrical machine, (ii) bearing rolling friction, and (iii) aerodynamic drag acting on the spinning rotor, also known as windage. In this study, the magnetic coupling allows the electrical machine to be completely decoupled from the rotor such that the electrical machine does not interact with the rotor, and associated losses thus decrease to zero. Therefore, bearing and aerodynamic losses can be characterized in an isolated manner at various vacuum levels. Table 2 provides an overview of the experimental plan.

Experiments were conducted by first setting the vacuum pressure in the housing to the specified level. Test vacuum pressures were within the range of medium (greater than $3 \mathrm{kPa}$ ) and rough vacuum ( $3 \mathrm{kPa}$ to $0.1 \mathrm{~Pa}$ ), corresponding to continuum and Knudsen flow regimes, which permits considering the air surrounding the rotor to obey continuum assumptions for analyses. 
Table 2. Experiment treatment combinations for all experiments conducted.

\begin{tabular}{cccl}
\hline Test Case & Motor Position & Vacuum Level (Pa (torr)) & $\begin{array}{c}\text { Vacuum Level and Flow } \\
\text { Regime }\end{array}$ \\
\hline 1 & Uncoupled & $27(0.2)$ & Medium vacuum, Knudsen \\
2 & & $67(0.5)$ & Medium vacuum, Knudsen \\
3 & & $133(1)$ & Rough vacuum, continuum \\
4 & & $1333(10)$ & Rough vacuum, continuum \\
5 & Coupled & $27(0.2)$ & Medium vacuum, Knudsen \\
6 & & $67(0.5)$ & Medium vacuum, Knudsen \\
7 & & $133(1)$ & Rough vacuum, continuum \\
\hline
\end{tabular}

Once the desired vacuum pressure was established, the electrical machine was energized until the rotor reached a maximum test velocity of $5000 \mathrm{rpm}\left(524 \mathrm{sec}^{-1}\right)$. Upon reaching the target speed, the system was allowed to spin freely until the stored energy was completely discharged. For experiments with the electrical machine in the uncoupled position, the electrical machine was decoupled from the rotor after reaching the target speed. Throughout the experiments, the rotor speed and housing vacuum pressure were monitored and recorded by the data acquisition system.

\section{Energy Storage and Discharge Models}

\subsection{Energy Storage and Loss}

The energy stored by the FESS is dependent on the rotor's moment of inertia, I, and the angular velocity, $\omega$. The moment of inertia is determined with:

$$
I_{\text {total }}=I_{\text {hub }}+\sum I_{\text {rim }}
$$

Considering each rim as a cylinder with an outer radius $r_{0}$, inner radius $r_{\mathrm{i}}$, and axial height $h$, the mass moment of inertia of each rim is found as:

$$
I_{\text {rim }}=\frac{1}{2} \rho_{\mathrm{m}} \pi h\left(r_{\mathrm{o}}^{4}-r_{\mathrm{i}}^{4}\right),
$$

where $\rho_{\mathrm{m}}$ is the material density. Similarly, the more complicated hub geometry, approximated as a thick-walled cylinder connected to a central thin disk, is given as:

$$
I_{\text {hub }}=\frac{1}{2} \rho_{\mathrm{m}} \pi\left(h\left(r_{\mathrm{o}}^{4}-r_{\mathrm{i}}^{4}\right)+h_{\mathrm{disk}} r_{\mathrm{i}}^{4}\right),
$$

where $h_{\text {disk }}$ is the thickness of the central part of the hub. The kinetic energy stored by the rotating rotor at any instant in time is therefore:

$$
E_{\text {rotor }}(t)=\frac{1}{2} I_{\text {total }} \omega(t)^{2} .
$$

Since the angular velocity of the rotor is monitored continuously throughout the passive discharge experiments, the stored energy can be found at any time during the experiment using Equation (4). Further, the energy loss due to passive discharge, $E_{\mathrm{PD}}$, for a given time interval is the difference between the initial energy, $E_{0}$, and the stored energy at a specific time, i.e.:

$$
E_{\mathrm{PD}}(t)=E_{0}-E_{\text {rotor }}(t),
$$

where $E_{0}=E_{\text {rotor }}(t=0)$. The energy loss due to passive discharge is further separated into the three primary sources considered in this study:

$$
E_{\mathrm{PD}}=E_{\mathrm{AD}}+E_{\mathrm{MB}}+E_{\mathrm{EM}}
$$

where $E_{\mathrm{AD}}, E_{\mathrm{MB}}$ and $E_{\mathrm{EM}}$ are the losses due to, correspondingly, the aerodynamic drag from air remaining in the housing, the rolling friction in the mechanical bearings, and the rotating 
electrical machine. The losses attributed to each source are discerned by systematically conducting experiments and evaluating collected test data.

The total energy loss during an instant of time is the passive discharge (rate of energy loss) as described in Equation (7). In this study, the passive discharge was approximated from test data using the difference quotient. Similar expressions can be derived for each of the above loss components:

$$
\frac{d E_{\mathrm{PD}}}{d t}=P_{\mathrm{PD}} \approx \frac{E_{\mathrm{PD}}\left(t_{2}\right)-E_{\mathrm{PD}}\left(t_{1}\right)}{t_{2}-t_{1}} .
$$

\subsection{Bearing Friction Losses}

The Hertzian rolling friction model is a well-established method for determining surface contact forces, as discussed in great detail in reference [12]. Other rolling friction models exist, see, e.g., [13], and are similar to the Hertzian contact model in that they use applied loads and material properties to determine rolling element contact area, deformation and friction. The Hertzian model is briefly discussed here, though a similar analysis could be performed with other models.

The applied loads, $F_{\text {load }}$, on the surfaces of the bearing rolling elements and races are used to analytically determine the contact area for each interface based on the deformation of the surfaces. The contact region in a ball bearing is defined as an elliptical region with a major radius $a$ and minor radius $b$. The normal stress at any point in the contact region is:

$$
F_{\mathrm{N}}=\frac{3 F_{\text {load }}}{2 \pi a b}\left[1-\left(\frac{x}{a}\right)^{2}-\left(\frac{y}{b}\right)^{2}\right]^{\frac{1}{2}} .
$$

From Equation (8), the friction force, $F_{\mathrm{F}}$, over the contact region of each ball is simply the integral of the normal force over the contact region times the friction coefficient $\mu$, i.e.:

$$
F_{\mathrm{F}}=\frac{3 F_{\text {load }} \mu}{2 \pi a b} \iint\left[1-\left(\frac{x}{a}\right)^{2}-\left(\frac{y}{b}\right)^{2}\right]^{\frac{1}{2}} d x d y .
$$

Thus, the passive discharge due to bearing friction is:

$$
P_{\mathrm{MB}}=\mu F_{\mathrm{N}} r \omega
$$

or:

$$
P_{\mathrm{MB}}=\mathrm{T}_{\mathrm{MB}} \omega,
$$

where torque $T_{\mathrm{MB}}$ is a function of the applied load (composed of radial and axial components), the friction coefficient, and the location of radial contact. In the current system, the applied load, $F_{\text {load }}$, is composed of the axial bearing preloads (from the partial rotor weight and spring washers acting on the lower and upper bearing, respectively) with lesser contributions from rotor precession and radial forces resulting from the magnetic thrust bearing misalignment. Assuming perfect alignment of the mechanical bearings and minimum loss conditions, i.e., uncoupled electrical machine and perfect vacuum, the energy loss directly related to the bearings follows a linear relation, indicated by Equation (11). While it is not possible to measure the loads in the current FESS, an approximately linear behavior associated with bearing losses facilitates separating air friction and bearing losses, which is discussed in greater detail in the following sections.

\subsection{Aerodynamic Drag Losses}

The analytical approach to determining the aerodynamic drag losses for a spinning rotor in rarefied gas is a non-trivial process dependent on the degree of rarefaction, mechanical properties of the gas, the geometry of the surface, and size of the environment as described by Shen [14]. These aspects affect the characteristic gradients, such as the air density and air velocity gradients, which cannot be measured by the current experimental 
setup. However, the theoretical approach presented by Broecker [15] is considered a more feasible approach to assessing aerodynamic drag losses. This model was designed for a disk rotating in a rarefied gas environment. Given the close resemblance of the conditions in a flywheel, Ertz [9] adopted this model. The general equation for the passive discharge loss to air friction is given as:

$$
P_{\mathrm{AD}}=M_{\text {total }} \omega,
$$

where $M_{\text {total }}$ is the drag moment.

The effects of rarefication on the frictional moment are considered in the following parameters: gas density, $\rho$, kinematic viscosity, $v$, and a reduced torque coefficient, $c_{\mathrm{m}}$. Ertz [9] adjusted Broecker's model [15] for the current system and assumed turbulent flow around the rotor at high velocity. Therefore, the frictional moment acting on the rotor is:

$$
M_{\text {total }}=M_{\text {top }}+M_{\text {bottom }}+M_{\text {side }}
$$

where $M_{\mathrm{top}}, M_{\mathrm{bottom}}$, and $M_{\text {side }}$ are the moments acting on the top, bottom, and outer diameter of the rotor. The top and bottom surfaces are congruent and can be found with,

$$
M_{\text {top }}=M_{\text {bot }}=\frac{1}{2} c_{\mathrm{m}} \operatorname{Re}^{\frac{1}{5}} \rho\left(r_{\mathrm{o}}^{\frac{23}{5}}-r_{\mathrm{i}}^{\frac{23}{5}}\right)\left(\frac{\mu}{\rho}\right)^{\frac{1}{5}} \omega^{\frac{9}{5}},
$$

where $R e$ is the Reynolds number given by Equation (15), and $\mu$ is dynamic viscosity. The torque coefficient $c_{\mathrm{m}}$ is dependent on the Reynolds number but can be thought of as approximately constant over the given velocity and pressure ranges [15]:

$$
R e=\frac{r_{\mathrm{O}}^{2} \omega \rho}{\mu} .
$$

Hence, the air friction moment equation can be rewritten, noting that many of the parameters are constant, yielding:

$$
M_{\mathrm{top}}=M_{\mathrm{bot}}=\frac{1}{2} c_{m} \rho r_{\mathrm{o}}^{\frac{2}{5}}\left(r_{\mathrm{o}}^{\frac{24}{5}}-r_{\mathrm{i}}^{\frac{23}{5}}\right) \omega^{2}=C_{1} \omega^{2},
$$

where $C_{1}$ is a constant. The air density is found with the ideal gas law,

$$
\rho=\frac{p M_{\mathrm{m}}}{R T},
$$

where $p$ is the air pressure, $M_{\mathrm{m}}$ is the molar mass of air, and $R$ and $T$ are the gas constant and temperature, respectively.

The frictional moment acting on the side of the rotor is defined as:

$$
M_{\text {side }}=2 \pi r_{o}^{2} h \tau \text {. }
$$

As shown by Broecker [15], $\tau$ can be derived as:

$$
\tau=\frac{M_{\mathrm{top}}}{2 \pi\left(\frac{5}{23}\right)\left(r_{o}^{3}-r_{i}^{3}\right)},
$$

i.e., $M_{\text {side }}$ is dependent on $M_{\text {top }}$, so it has the same form as $M_{\text {top }}$, meaning the frictional moment is of the form,

$$
M_{\text {total }}=2 C_{1} \omega^{2}+C_{2} \omega^{2}=C_{\mathrm{AD}} \omega^{2},
$$

where $C_{2}$ and $C_{\mathrm{AD}}$ are constants. Finally, combining Equations (12) and (20) yields,

$$
P_{\mathrm{AD}}=C_{\mathrm{AD}} \omega^{3} \text {. }
$$


Given that the rotor geometry does not change during an experiment and the vacuum pressure is constant, the passive discharge due to aerodynamic drag increases cubically with angular velocity. Therefore, with the electrical machine in the uncoupled position, aerodynamic drag losses can be identified as a cubic term in a relation analogous to Equation (6).

\subsection{Electrical Machine Losses}

The electrical machine only affects passive discharge losses when in the coupled position. When coupled, the employed electrical machine can operate (i) as a motor, adding energy to the system; (ii) as a generator, removing energy; (iii) or in standby mode, doing neither. The latter case (no-load operation) is herein considered. The electrical machine passive discharge is found as the difference between the passive discharge while the motor is in the coupled and uncoupled positions, i.e.,

$$
P_{\mathrm{EM}}=P_{\text {PD.coupled }}-P_{\text {PD.uncoupled }} \text {. }
$$

The electrical machine passive discharge is characterized by the aerodynamic drag in the electrical machine $\left(P_{\mathrm{EM} . \mathrm{AD}}\right)$, which is needed for cooling, and rolling friction $\left(P_{\mathrm{EM} . \mathrm{MB}}\right)$ from the machine's bearings, as per analyses akin to the previous sections. $P_{\mathrm{EM}}$ further includes eddy-current and hysteresis losses $\left(P_{\text {core }}\right)$ and permanent magnet losses $\left(P_{\text {pm }}\right)[16]$, hence:

$$
P_{\mathrm{EM}}=P_{\mathrm{EM} . \mathrm{AD}}+P_{\mathrm{EM} . \mathrm{MB}}+P_{\text {core }}+P_{\mathrm{pm}} .
$$

The eddy-current and hysteresis losses are described as,

$$
P_{\text {core }}=K_{\mathrm{h}} B^{2} f+K_{\mathrm{e}} V_{\mathrm{c}} B^{2} f^{2},
$$

where $K_{\mathrm{h}}$ and $K_{\mathrm{e}}$ are constants determined from material and machine parameters, $V_{\mathrm{c}}$ and $B$ are the volume of the ferromagnetic core and magnetic peak flux density, respectively, and $f$ is the frequency of magnetization, which is proportional to the machine angular velocity.

The losses associated with the permanent magnets can be found with,

$$
P_{\mathrm{pm}}=K_{\mathrm{pm}} V_{\mathrm{pm}} B^{2} f^{2},
$$

where $K_{\mathrm{pm}}$ is again a constant determined from material and machine parameters and $V_{\mathrm{pm}}$ is the magnet volume. Further details on the various parameters in Equations (24) and (25) can be found in reference [16]. While the parameters associated with the electrical machine used in the experiments are unknown, Equations (23) to (25) expose the relationships considering the key constants associated with the electrical machine passive discharge, yielding the form of a third-order polynomial as shown in Equation (26), where $T_{\mathrm{EM}}$ is related to bearing friction, $C_{\mathrm{EM} 1}$ and $C_{\mathrm{EM} 2}$ with electromagnetic phenomena, and $C_{\mathrm{EM}} \mathrm{AD}$ to aerodynamic drag. The constants related to bearing friction and aerodynamic drag are found akin to the derivation made for Equations (11) and (21).

$$
P_{\mathrm{EM}}=T_{\mathrm{EM}} \omega+C_{\mathrm{EM} 1} \omega+C_{\mathrm{EM} 2} \omega^{2}+C_{\mathrm{EM} \cdot \mathrm{AD}} \omega^{3}
$$

\section{Results and Discussion}

In the present study, the characterization of FESS losses commences with identifying the minimum loss case, i.e., the experimental condition with the lowest losses as per Table 2, herein termed the baseline case. Then, the passive discharge to aerodynamic drag is separated from the baseline case based on the relationships established in the previous sections. Finally, with baseline losses and aerodynamic drag characterized, the electrical machine losses are examined. 


\subsection{Baseline Losses}

The baseline losses are characterized by treatment combinations \#1 to \#4 in Table 2, which has the electrical machine decoupled such that $P_{\mathrm{EM}}=0$. In the following, the vacuum levels of $27 \mathrm{~Pa}, 67 \mathrm{~Pa}, 133 \mathrm{~Pa}$, and $1333 \mathrm{~Pa}$ are referred to as the minimum, low, intermediate, and high-pressure states, respectively. The average total discharge time and half-life time, i.e., the point at which half the kinetic energy is discharged, are summarized in Table 3. It can be observed from the data that neither half-life time nor total discharge time correlates directly with the magnitude of the applied pressure, i.e., a lower pressure did not necessarily produce a longer half-life and total discharge time. The reasons for this behavior are discussed in the following.

Table 3. Rotor kinetic energy half-life time and total discharge time for all pressure states with the electrical machine decoupled.

\begin{tabular}{cccc}
\hline Test Case & Vacuum Level (Pa (torr)) & Half-Life Time (min) & Total Discharge Time (min) \\
\hline 1 & $27(0.2)$ & 37 & 276 \\
2 & $67(0.5)$ & 49 & 450 \\
3 & $133(1)$ & 55 & 357 \\
4 & $1333(10)$ & 20 & 250 \\
\hline
\end{tabular}

Recall that the velocity of the rotor was monitored continuously during testing, allowing the stored energy to be calculated with Equation (5) and the passive discharge to be found as the difference quotient as shown in Equation (7). The passive discharge is plotted against angular velocity in Figure 3 to facilitate comparisons between the different test cases. Since passive discharge results are relatively noisy after differentiation due to random errors during testing, three repetitions of each test case were performed and averaged together in an attempt to minimize data scatter.

To identify the minimum loss case, the peak passive discharge data were computed for each vacuum pressure state at four-rotor angular velocities, i.e., $500 \mathrm{rpm}\left(52 \mathrm{sec}^{-1}\right)$, $1000 \mathrm{rpm}\left(105 \mathrm{sec}^{-1}\right), 2500 \mathrm{rpm}\left(262 \mathrm{sec}^{-1}\right)$, and $5000 \mathrm{rpm}\left(524 \mathrm{sec}^{-1}\right)$. Data for peak passive discharge are plotted against pressure in Figure 4. Notably, the lowest passive discharge, i.e., the baseline case, was consistently identified to occur for the intermediate vacuum level of $133 \mathrm{~Pa}$, while passive discharge increased again for the lower tested pressure states. This behavior, which also affected the half-life and total discharge times shown in Table 3, is not intuitive and was initially believed to be caused by a failure of the instrumentation. However, the careful investigation of the equipment indicated that the sensors were functioning properly. In addition, pressure-induced deformation of the FESS housing and thus possible effects on bearing friction are considered negligible for the comparatively minuscule differences in applied pressures. Specifically, considering loads imposed on the housing by evacuating it from atmospheric pressure $(101.3 \mathrm{kPa})$ to the applied vacuum levels, the difference in loads acting on the housing is about $0.1 \%$ between the minimum (27 Pa) and intermediate (133 Pa) pressure states. It can also be speculated that the observed rise in passive discharge at the lower vacuum levels is related to the change in the flow regime in the vacuum transition region with diminishing continuum gas properties and potential effects on the effectiveness of the employed vacuum pump. However, the exact cause for the observed effect and potential remedies are unknown at this point and remain the subject of future work. Hence, for this study, results for the minimum (27 Pa) and low (67 Pa) pressure states are excluded from subsequent analyses and discussion. 


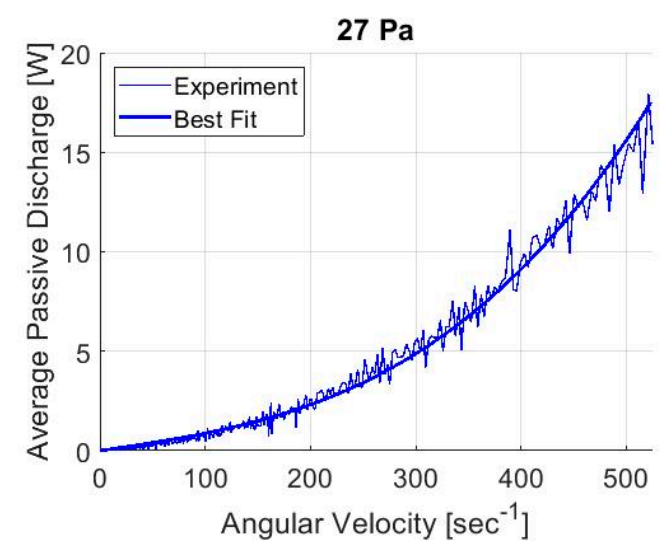

(A)

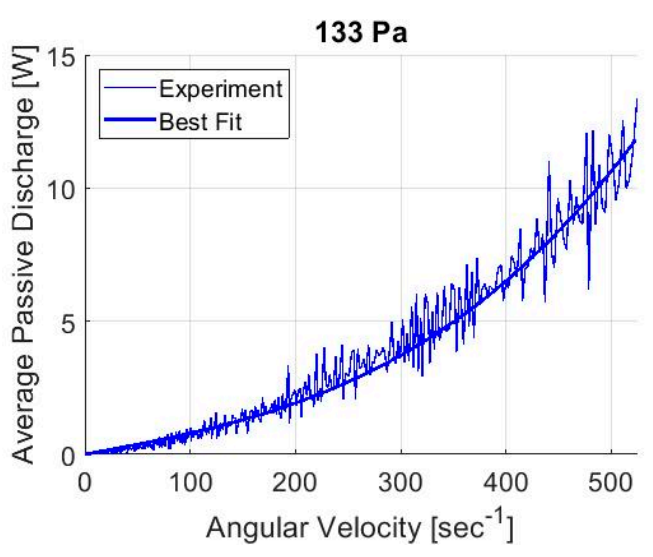

(C)

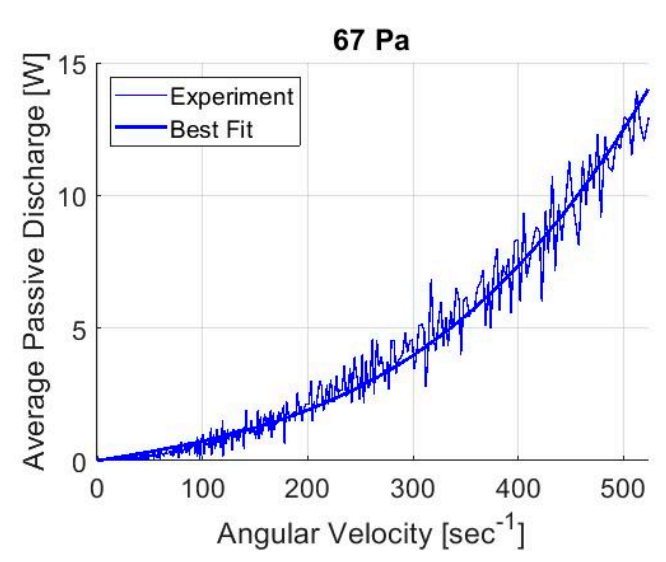

(B)

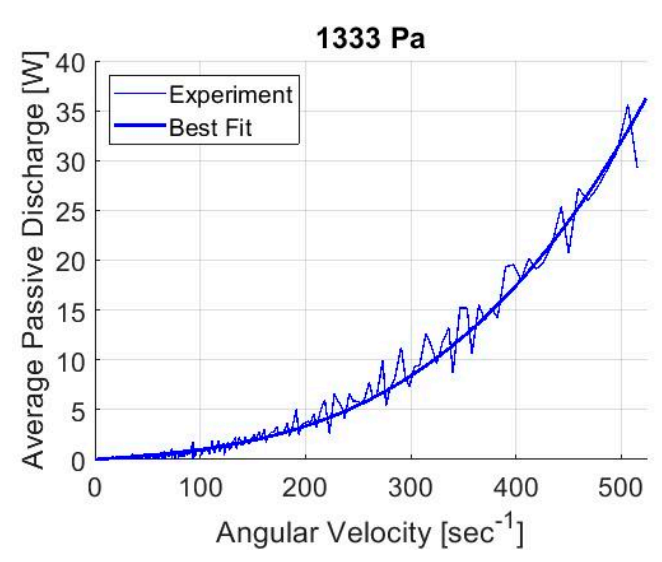

(D)

Figure 3. Average passive discharge and best-fit approximations for the four tested vacuum levels: (A) minimum, (B) low, (C) intermediate, and (D) high-pressure.

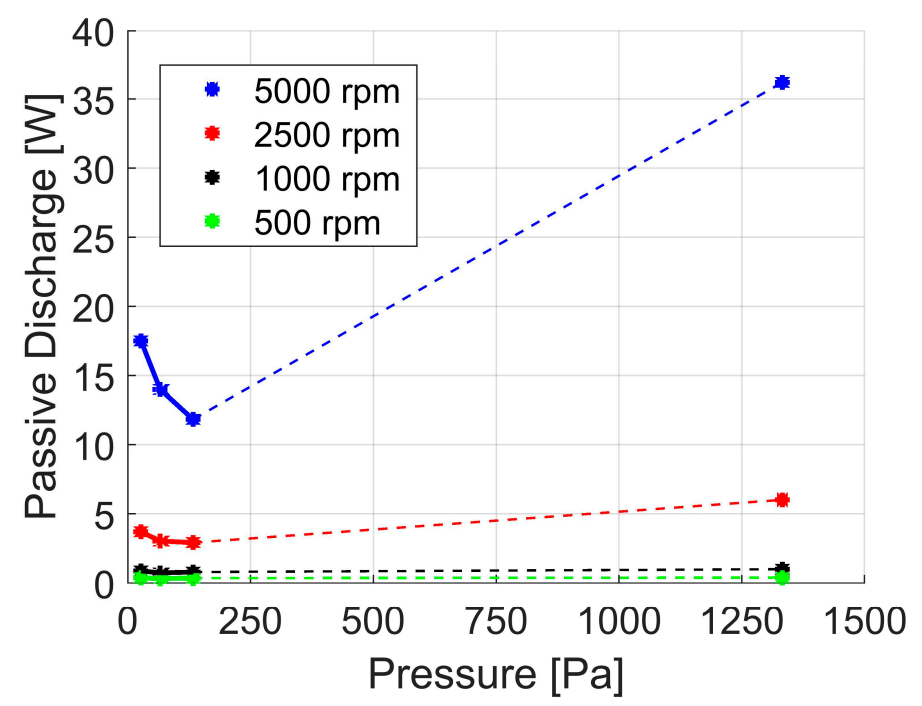

Figure 4. Total rotor passive discharge versus pressure at four distinct rotor speeds. Dashed lines are included to indicate trends in the measured data. 


\subsection{Discerning Sources of Passive Discharge}

Best fit approximations of the test data were computed, as shown in Figure 3. Considering Equations (6), (7), (11), and (21), and recalling the electrical machine was decoupled, the best-fit equation can be defined as

$$
P_{\mathrm{PD}}=\alpha_{1} \omega+\alpha_{2} \omega^{3}
$$

where $\alpha_{1}$ and $\alpha_{2}$ are constant coefficients that define bearing rolling friction and aerodynamic drag, equivalent to $T_{\mathrm{MB}}$ from Equation (11) and $C_{\mathrm{AD}}$ from Equation (21), respectively. The curve fitting tool in the numerical computing environment MATLAB was used (MathWorks, Natick, MA, USA). Specifically, the robust nonlinear least-squares regression method was employed as it uses the least absolute residuals (LAR) method, which minimizes the absolute difference of the residuals to minimize the influence of outliers. This method is preferred when the data have few outliers [17], as shown in Figure 3, where data are noisy but contain few outliers. Hence, the LAR method is considered appropriate.

From Equation (27), the bearing friction and drag components can be separated, and the magnitude of energy losses to each component can be clearly identified. Considering the baseline case (intermediate vacuum level) and the high-pressure state, the coefficients were computed as listed in Table 4 along with goodness of fit statistical parameters (SSE: error sum of squares, Adj-R2: adjusted R-squared, RMSE: root-mean-squared error). The statistical parameters indicate that the best-fit approximations accurately represent each dataset, suggesting the best-fit approximations are appropriate for modeling and predictions. Specifically, SSE values indicate small random error effects, and values for Adj-R2 and RMSE approaching unity and zero confirm a good fit and small standard deviation, respectively.

Table 4. Best fit coefficients for the baseline and high-pressure test cases along with goodness of fit statistical parameters (SSE: error sum of squares, Adj-R2: adjusted R-squared, RMSE: root-meansquared error).

\begin{tabular}{ccc}
\hline Parameter & Baseline Case (133 Pa) & High-Pressure (1330 P) \\
\hline$\alpha_{1} \equiv T_{\mathrm{MB}}$ & $0.0074 \mathrm{Ws}$ & $0.0078 \mathrm{Ws}$ \\
$\alpha_{2} \equiv C_{\mathrm{AD}}$ & $5.536 \times 10^{-8} \mathrm{Ws}^{3}$ & $2.238 \times 10^{-7} \mathrm{Ws}^{3}$ \\
SSE & 1.006 & 1.809 \\
Adj-R2 & 0.9995 & 0.9997 \\
RMSE & 0.0529 & 0.0852 \\
\hline
\end{tabular}

The rolling friction coefficients shown in Table 4 are nearly equal, i.e., they are within approximately $5 \%$ of each other. This finding indicates that rolling friction is indeed independent of pressure. It further suggests that the assumptions and derivation made for establishing Equation (11) are proper for describing this loss component.

In terms of aerodynamic drag losses, the corresponding best-fit coefficients increase by nearly an order of magnitude between the baseline and high-pressure cases (i.e., $5.536 \times 10^{-8} \mathrm{Ws}^{3}$ versus $2.238 \times 10^{-7} \mathrm{Ws}^{3}$ ). When deriving Equation (16), it was discussed that the torque coefficient, $c_{\mathrm{m}}$, is approximately constant within a small pressure range. Therefore, only the air density $\rho$, is nonconstant, that is, density varies linearly with pressure according to the ideal gas law, Equation (17). Hence, considering the aerodynamic drag coefficient to be a function of pressure, as in Equation (28), the linear term shown in Equation (29) can be assumed for approximating aerodynamic drag for pressures above $133 \mathrm{~Pa}$.

$$
\begin{gathered}
P_{\mathrm{AD}}=\alpha_{2}(p) \omega^{3} \\
P_{\mathrm{AD}}=\left(1.404 \times 10^{-10} p+3.664 \times 10^{-8}\right) \omega^{3}
\end{gathered}
$$

where $P_{\mathrm{AD}}, p$ and $\omega$ are in $\mathrm{Ws}, \mathrm{Pa}$ and $\sec ^{-1}$, respectively. 
The various components of passive discharge are predicted and plotted in Figure 5 for the baseline case. As expected, bearing losses are the largest source of passive discharge at low rotor speeds where aerodynamic drag is small. The latter dominates losses at higher rotor speeds. The point where aerodynamic drag exceeds bearing friction is 3486 $\mathrm{rpm}\left(365 \mathrm{sec}^{-1}\right)$. Aerodynamic drag is usually considered the most significant source of passive discharge due to the sustained rotor speed being quite high in FESS. Nevertheless, the analysis confirms that passive discharge due to bearing friction is not insignificant for low-cost FESS systems. In fact, bearing losses can be a significant source of passive discharge even in FESS with magnetic bearings if regular deep discharge situations occur. Magnetic bearings rely on eddy currents to maintain stability, requiring a larger magnetic field to stabilize the rotor at lower rotor speeds [18].

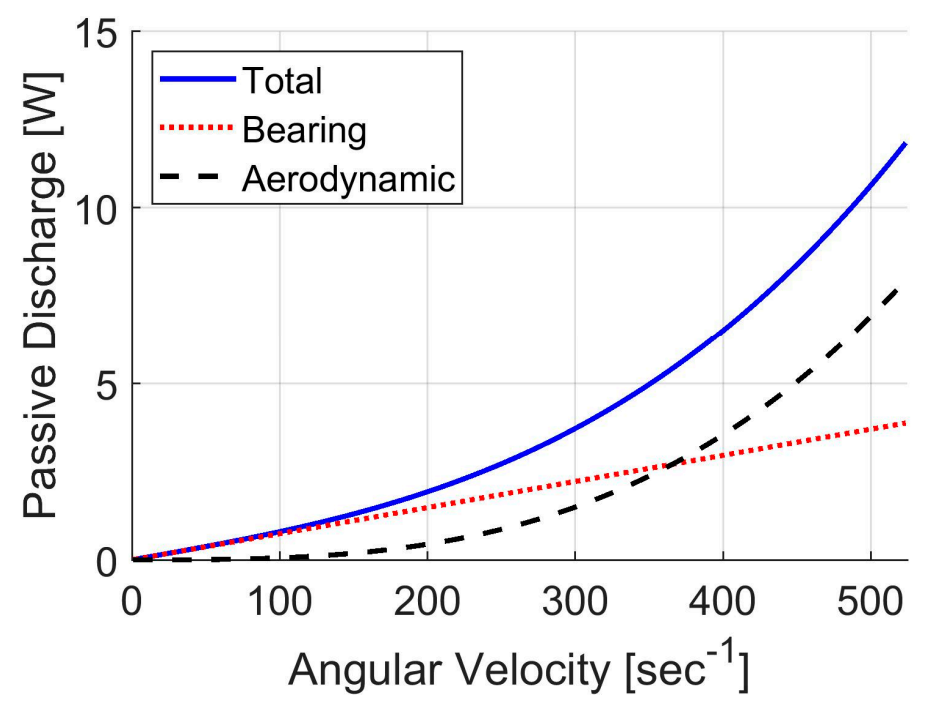

Figure 5. Predictions of the total rotor passive discharge and the components related to aerodynamic and bearing losses for the baseline case (intermediate vacuum level).

When coupled to the FESS, the electrical machine introduces its own passive discharge, from forced air cooling, bearing friction, and core and permanent magnet losses, as discussed above. These sources are collectively discussed because, as demonstrated by Equation (26), it is not possible to decouple each source of passive discharge losses while operating the electrical machine in standby mode. The data for this analysis is collected from treatment case \#7 in Table 2. Subtracting the baseline losses (treatment case \#3) from these data yields the passive discharge caused by the electrical machine. Given that Equation (26) includes linear and higher-order polynomials, it is interesting to again apply Equation (27) to generate an approximation for the electrical machine losses. In this approach, the linear and cubic terms collectively represent mechanical and electromagnetic loss sources, i.e., the linear term captures bearing rolling friction and core losses, while the cubic term represents the aerodynamic drag and the losses associated with the permanent magnets. The experimental data and best-fit results are depicted in Figure 6, and the coefficients $\alpha_{1}$ and $\alpha_{2}$ are listed in Table 5 . The close congruence between the curves in the graph in Figure 6 and the goodness of fit statistical parameters in Table 5 indicate that Equation (27) is an adequate means for creating a passive discharge approximation for the electrical machine.

The total passive discharge and its components for the electrical machine (no-load) and mechanical losses as per the baseline case are depicted in Figure 7. Clearly, the electrical machine used in this experimental setup is the largest source of passive discharge by a significant margin. From a design point of view, having the electrical machine operate outside the vacuum housing, while being a valuable feature for this study, is highly detrimental to the performance of the FESS when continual charge and discharge operations 
are performed, requiring the electrical machine to be permanently coupled to the rotor. Besides including the electrical machine in the vacuum housing, the machine can be designed to minimize mechanical losses, such as through integration with the rotor itself [19]. While the machine would still suffer losses due to electromagnetic effects, its total contribution to losses would be minimized. For intermittent charge and discharge operations, however, the present FESS design is attractive for its ability to nearly eliminate electrical machine losses (due to its rotor decoupling feature) while also allowing for easy cooling of the electrical machine in high-power transfer operations.

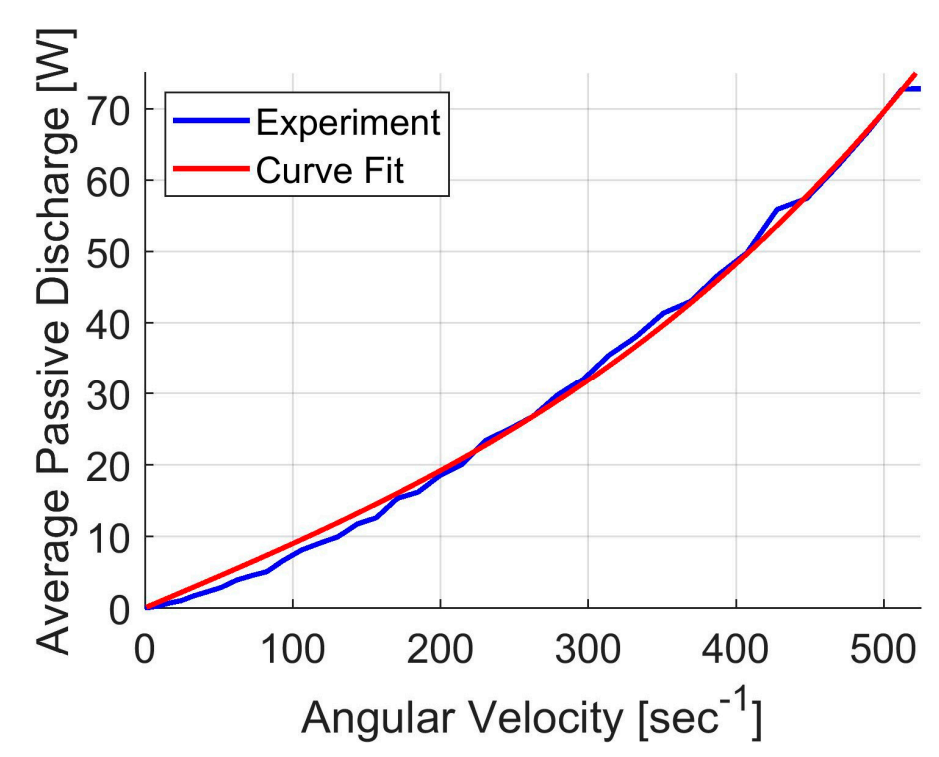

Figure 6. Experimental data and curve fit approximation for the electrical machine passive discharge for the baseline pressure state (intermediate vacuum level).

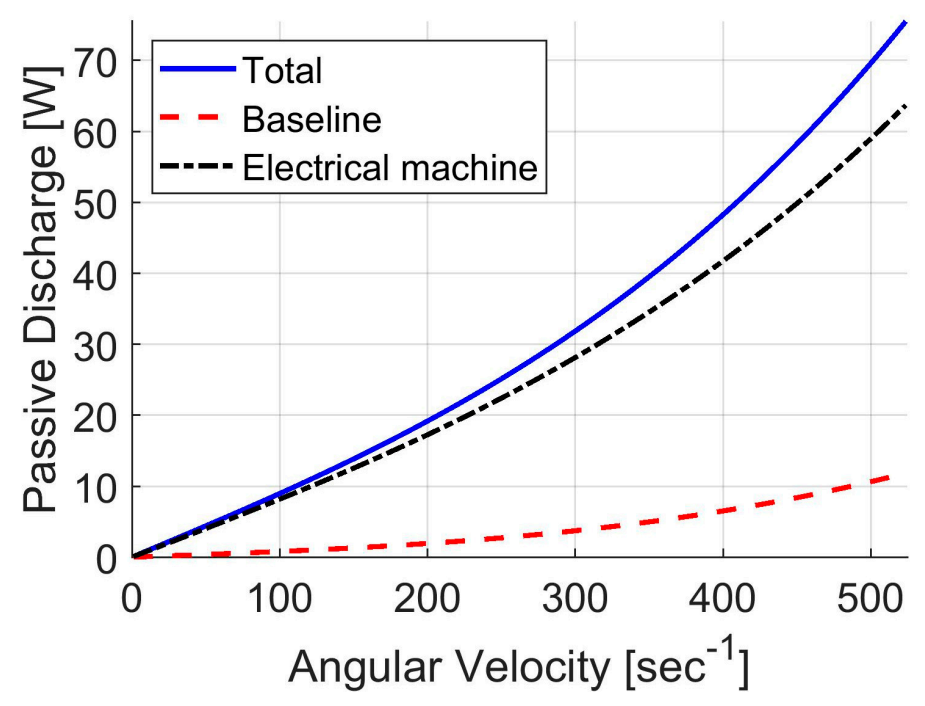

Figure 7. Predictions of the combined total passive discharge for the rotor with the coupled electrical machine, as well as the components related to the electrical machine no-load losses and the baseline case losses (intermediate vacuum level, electrical machine decoupled). 
Table 5. Best fit coefficients for the electrical machine passive discharge approximation along with goodness of fit statistical parameters (SSE: error sum of squares, Adj-R2: adjusted R-squared, RMSE: root-mean-squared error).

\begin{tabular}{cc}
\hline Parameter & Baseline Case (133 Pa) \\
\hline$\alpha_{1}$ & $0.0864 \mathrm{Ws}$ \\
$\alpha_{2}$ & $2.004 \times 10^{-7} \mathrm{Ws}^{3}$ \\
SSE & 4.058 \\
Adj-R2 & 0.9999 \\
RMSE & 0.2443 \\
\hline
\end{tabular}

\section{Conclusions}

Low-cost, small-scale flywheel energy storage systems, such as those suitable for residential, small-scale commercial and transportation applications, require managing tradeoffs between cost, size, weight and performance. It is therefore important to understand the effects that design parameters have on energy losses and thus passive discharge, which can primarily be attributed to bearing rolling friction, aerodynamic drag on the flywheel rotor, and the electrical machine employed for transferring energy to and from the rotor.

In the present study, passive discharge experiments were performed with an actual flywheel energy storage device to measure passive discharge at different vacuum pressures in the flywheel housing. Vacuum pressures corresponding to continuum and Knudsen flow regimes were applied in this study. The flywheel device permits the electrical machine to be fully decoupled from the rotor, thereby enabling the study of the individual loss sources. Analytical models relating to each passive discharge source were developed, yielding a relatively straightforward set of equations that allowed discerning the loss components from the experimental data. These findings are considered a valuable contribution to the development and improvement of modeling approaches to be employed in the design and optimization of flywheel systems.

The analyses revealed that bearing rolling friction was consistent across all experiments (i.e., vacuum pressures), with the related passive discharge increasing linearly with rotor speed. The study further confirmed that aerodynamic drag on the rotor is a major source of passive discharge, even in a medium vacuum environment, as passive discharge increases cubically with rotor speed. Electrical machine losses were found to be most significant for the FESS design used in this study. These findings lead to the recommendation to reduce electrical machine losses by integrating the machine into the vacuum housing and possibly even the flywheel rotor itself. For intermittent charge and discharge operations, however, the present FESS design with its ability to decouple the rotor from the electrical machine is attractive since electrical machine losses can be eliminated when the machine is in standby mode.

Author Contributions: Conceptualization and methodology, M.S. and P.M.; validation, formal analysis, investigation, M.S.; resources, P.M.; data curation, M.S.; writing—original draft preparation, M.S.; visualization, writing — review and editing, M.S. and P.M.; supervision, project administration, P.M.; funding acquisition, P.M. All authors have read and agreed to the published version of the manuscript.

Funding: This research was funded by the Natural Science and Engineering Research Council of Canada (NSERC) Energy Storage Technology Network and the Canada First Research Excellence Fund with grant number Future Energy Systems T06-P03. The APC was funded by the Canada First Research Excellence Fund with grant number Future Energy Systems T06-P03.

Acknowledgments: The author would like to thank Bernie Faulkner for his technical support for performing the experimental work.

Conflicts of Interest: The authors declare no conflict of interest. 


\section{References}

1. Dunn, B.; Kamath, H.; Tarascon, J.M. Electrical storage for the grid: A battery of choices. Science 2011, 334, 928-935. [CrossRef] [PubMed]

2. Luo, X.; Wang, J.; Dooner, M.; Clark, J. Overview of current development in electrical energy storage technologies and application potential in power system operation. Appl. Energy 2015, 137, 511-536. [CrossRef]

3. Hadjipaschalis, I.; Poullikkas, A.; Efthimiou, V. Overview of current and future energy storage technologies for electric power applications. Renew. Sistain. Energy Rev. 2009, 13, 1513-1522. [CrossRef]

4. Mulcahy, T.; Hull, J.; Uherka, K.; Abbound, R.; Juna, J. Test results of 2-kWh flywheel using passive PM and HTS bearings. IEEE Trans. Appl. Supercond. 2001, 11, 1729-1739. [CrossRef]

5. Schulz, A.; Sima, S.; Hinterdorfer, T.; Wassermann, J. Innovative Schwungradspeicher mit hoher Energieeffizienz und Zuverlässigkeit. Elektrotech. Informationstechnik 2015, 132, 481-490. [CrossRef]

6. Coombs, T.A.; Campbell, A.M.; Cardwell, D.A. Development of an active superconducting magnetic bearing. IEEE Trans. Appl. Supercond. 1995, 5, 630-633. [CrossRef]

7. Xia, Z.; Chen, Q.Y.; Ma, K.B.; McMichael, C.K.; Lamb, M.; Cooley, R.S.; Fowler, P.C.; Chu, W.K. Design of superconducting magnetic bearings with high levitating force for flywheel energy storage systems. IEEE Trans. Appl. Supercond. 1995, 5, 622-625. [CrossRef]

8. Filatov, A.V.; Maslen, E.H. Passive magnetic bearing for flywheel energy storage systems. IEEE Trans. Magn. 2001, 37, 3913-3924. [CrossRef]

9. Ertz, G. Development, Manufacturing and Testing of a Multi-Rim (Hybrid) Flywheel Rotor. Master's Thesis, Leibniz University Hannover, Hannover, Germany, 2014.

10. Ha, S.K.; Han, H.H.; Han, H.Y. Design and manufacture of a composite flywheel press-fit multi-rim rotor. J. Reinf. Plast. Comp. 2008, 27, 953-965. [CrossRef]

11. Krack, M.; Secanell, M.; Mertiny, P. Cost optimization of hybrid composite flywheel rotors for energy storage. Struct. Multidiscip. Optim. 2010, 41, 779-795. [CrossRef]

12. Harris, T.A.; Kotzalas, M.N. Advanced Concepts of Bearing Technology, 5th ed.; CRC Press: Boca Raton, FL, USA, 2006.

13. Flom, D.G.; Bueche, A.M. Theory of rolling friction for spheres. J. Appl. Phys. 1959, 30, 1725-1730. [CrossRef]

14. Shen, C. Rarefied Gas Dynamics; Spring: Berlin, Germany, 2005.

15. Broecker, V.E. Theorie und Experiment zum Reibungswiderstand der glatten rotierenden Scheibe bei tubolenter Strömung. Z. Angew. Math. Mech. 1959, 39, 68-76. [CrossRef]

16. Tong, W. Mechanical Design of Electric Motors; CRC Press: Boca Raton, FL, USA, 2014.

17. Least-Squares Fitting. Available online: http://www.mathworks.com/help/curvefit/least-squares-fitting.html (accessed on 6 November 2020).

18. Traxler, A. Losses in Magnetic Bearings. In Magnetic Bearings; Maslen, E.H., Schweitzer, G., Eds.; Spring: Berlin, Germany, 2009; pp. 135-150.

19. Amiryar, M.E.; Pullen, K.R. A review of flywheel energy storage system technologies and their applications. Appl. Sci. 2017, 7, 286. [CrossRef] 